\title{
A review on Hydroxamic Acids: Widespectrum Chemotherapeutic Agents
}

\author{
Zainab Syed ${ }^{1,2}$, Kumar Sonu $^{1}$, Aman Dongre ${ }^{1,2}$, Gopesh Sharma ${ }^{2}$, Monika Sogani ${ }^{*}$ \\ ${ }^{1}$ Schoolof Civil and Chemical Engineering, Manipal University Jaipur, Rajasthan 300307, India \\ ${ }^{2}$ Department of Biosciences, Manipal University Jaipur, Rajasthan 300307, India \\ *Author for correspondence: Tel: +919414207450
}

Received: January 28, 2020. Revised: March 10, 2020. 2nd Revised: May 25, 2020.

Accepted: June 22, 2020. Published: July 20, 2020.

\begin{abstract}
Recent developments in drug discovery have highlighted the ability of hydroxamic acids to form complexes with various metal ions, in particular iron, zinc, magnesium and calcium, and this imparts them with a number of unique biological and pharmacological properties. This review provides information on the most significant developments of the hydroxamate compounds in the medicinal area with a focus on Suberoylanilide hydroxamic acid (SAHA) and its derivatives with antibacterial, antifungal, antiviral, antimalarial, antitubercular and anticancer effect and their possible molecular mechanisms. One of the latest favorable developments has been on developing SAHA and its hybrids as potent anti-tuberculosis drugs. Moreover, the combination of the hydroxamic acids with other pharmacophore groups in one molecule can lead to the creation of a huge number of new compounds with promising properties as a multifunctional drug. Also, within the last decade, active research has focused to create these compounds into a class of new antiviral drugs designed to treat influenza, hepatitis $\mathbf{C}$, and other viral infections. Thus, both linear hydroxamic acids and cyclic acids having the uniqueness of the set of bioactive properties, have been compiled here and could be looked forward as the basis for the development of effective drugs and forpilot scale productions through nitrile and amides via chemical and biological transformations using amidase and acyltransferase enzymes. Such synthesis of medicinally significant compounds from amide containing waste derived feedstocks using microbial agents is also a key factor for stimulating bioeconomy.
\end{abstract}

Keywords: Hydroxamic acids, biological activities, antimicrobial, anti-inflammatory, anti-cancer, anti-malarial, anti-tubercular, anti-oxidant.

\section{INTRODUCTION}

Hydroxamic acids and their derivatives have attracted considerable attention, due to their pharmacological, toxicological and pathological properties. They have a wide spectrum of activities in all types of biological systems and are structural subunits of various biologically active natural products and are valuable synthetic precursors and pharmaceuticals. They can also be used as peptide deformilases or enzyme inhibitors [1]. Various substituted analogues of hydroxamic acid are reported to possess antiinflammatory, anti-tubercular, anti-leukemic, anti-microbial and tumor inhibiting properties. They play a vital role in the manufacturing of various biologically active drugs as antidepressants, anti-tumor agents, anti-HIV agents and antimalarial agents and are also used in industries as insecticides, antioxidants, as inhibitors of corrosion and for the extraction of toxic elements [2].

\section{BIOLOGICAL AND MEDICINAL SIGNIFICANCE OF HYDROXAMIC ACIDS}

Although they have been known from long ago to be biologically active, their varied biological features are still of great scientific interest. Given below is a brief account of various alterations conducted hydroxamic acid molecules and their associated biological activities.

\section{A. Antimicrobial activity}

\section{Antibacterial activity}

Bacterial stalk rot of maize is caused by a certain strain of Erwinia chrysanthemi. Corcuera et al.[3] proposed 2,4Dihydroxy-7-methoxy-1,4-benzoxazin-3-one (DIMBOA), to be involved in the resistance towards Erwinia species as shown in Fig. 1. They reported that DIMBOA inhibits the growth of several soft rotting Erwinia species at concentrations of $0.2-0.3 \mathrm{mM}$ and that strains nonpathogenic to maize were more sensitive to DIMBOA than pathogenic strains.



Fig. 1. Chemical structure of DIMBOA (2, 4-dihydroxy-7methoxy, 1,4- benzoxazin-3-one)

Wang et al. [4] investigated the mutagenic and antibacterial activity of several hydroxamic acids with differing acyl groups as shown in Fig. 2. They carried the 
Ames spot test for studying chemical mutagenicity of different hydroxamic acids as Benzoylhydroxamic acid, salicyl hydroxamic acid, indole-2-carbohydroxamic acid, pButoxyphenylacetohydroxamic Naphthoylhydroxamic acid acid,

2-

Benzoylaminoacetohydroxamic acid andas well as t anti-bacterial effects onStreptococcus faecalis, Escherichia coli, Bacillus cereus, Proteus morganii and Salmonella typhimurium.

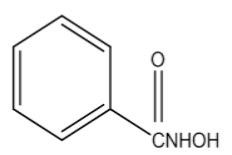

Benzoylhydroxamic acid

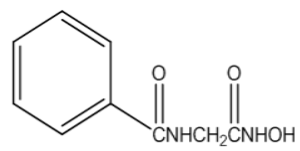

Benzoylaminoacetohydroxamic acid



Indole-2-carbohydroxamic acid

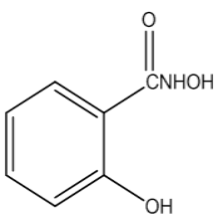

Salicylhydroxamic acid

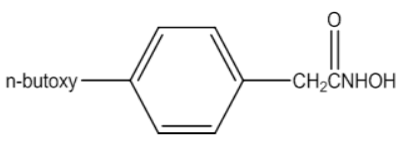

p-Butoxyphenylacetohydroxamic acid

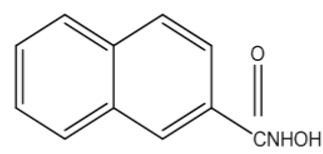

2-Naphthoylhydroxamic acid
Fig. 2. Hydroxamic acids showing antibacterial activity

All the hydroxamic acids tested except pButoxyphenylacetohydroxamic acid produced clear antibacterial zones and mutations in both $S$. typhimurium strains TA100 and TA98. It appears that these mutagenic hydroxamic acids may interact with bacterial deoxyribonucleic acid. 2-Naphthoylhydroxamic acid was effective against Streptococcus faecalis and Escherichia coli and was slightly effective against Bacillus cereus and Proteus morganii. Benzoylhydroxamic acid, salicylhydroxamic acid, and indole-2-carbohydroxamic acid were slightly effective against some bacterial strains. pButoxyphenylacetohydroxamic acid lacked mutagenicity for $S$. typhimurium and was virtually ineffective against the tested bacteria. Thus, hydroxamic acid function appears to be essential for the mutagenic and antibacterial activities of hydroxamic acids, whereas the acyl moiety appears to determine their potency. Although hydroxamic acids have been shown to be effective inhibitors of bacterial and plant urease [5], their antibacterial activity probably is not due to urease inhibition [6]. It seems likely that the antibacterial activity is due to their interaction with bacterial deoxyribonucleic acid since hydroxamic acids in earlier various researches have proven to show both mutagenic and antibacterial effects.

Kaushal et al. [7] studied the antibacterial activity of five structurally related titanium (IV) heteroleptic complexes, $\left[\mathrm{TiCl}_{2}(\mathrm{bzac})(\mathrm{L} 1-4)\right]$ and $\left[\mathrm{TiCl}_{3}(\mathrm{bzac})(\mathrm{HL} 5)\right]$; bzac $=$ benzoylacetonate; L1-5 = benzohydroximate (L1), salicylhydroximate (L2), acetohydroximate (L3), hydroxyurea (L4), and N-benzoyl-N-phenyl hydroxylamine (L5), against ten pathogenic bacterial strains. The investigated microorganisms were E.aerogenes MTCC 6128, Micrococcus luteus MTCC 1809, Staphylococcus aureus MTCC 3160, Staphylococcus epidermidis MTCC3086, Aeromonas hydrophila MTCC 1739, Alcaligenes faecalis MTCC 126, Shigella sonnei MTCC 2957, Klebsiella pneumonia MTCC 3384, Pseudomonas aeruginosa MTCC 1035, and Salmonella typhimurium MTCC 1253. On the basis of the zone inhibition it was observed that complex $\left[\mathrm{TiCl}_{2}(\mathrm{bzac})(\mathrm{L} 1)\right]$ was more potent against $S$. sonnei with inhibition zone of diameter $13.5 \mathrm{~mm}$ and $\left[\mathrm{TiCl}_{2}(\mathrm{bzac})(\mathrm{L} 2)\right]$ was more active against $A$. hydrophila with inhibition zone of diameter $18 \mathrm{~mm}$ among all the bacterial strains. Complex $\left[\mathrm{TiCl}_{2}\right.$ (bzac)(L3)]was found to be more active against $E$. aerogenes, $S$. epidermidis, and $S$. sonnei with inhibition zone of diameter $13 \mathrm{~mm}, 13 \mathrm{~mm}$, and $14.5 \mathrm{~mm}$, respectively. Complex $\left[\mathrm{TiCl}_{2}(\mathrm{bzac})(\mathrm{L} 4)\right]$ was more potent against $S$. typhimurium, E. aerogenes, S.epidermidis, S. aureus, and M. luteus with $13 \mathrm{~mm}, 13 \mathrm{~mm}$, $15 \mathrm{~mm}, 15 \mathrm{~mm}$, and $16.5 \mathrm{~mm}$ zone inhibition diameters, respectively, and complexes $\left[\mathrm{TiCl}_{3}\right.$ (bzac)(HL5)] were found to be more effective against $P$. aeruginosa, $S$. aureus, and $A$. faecalis with $17 \mathrm{~mm}, 16 \mathrm{~mm}$, and $14 \mathrm{~mm}$ zone inhibition diameters, respectively. On the basis of the Minimal Inhibitory Concentration (MIC) values it was found that complex, $\left[\mathrm{TiCl}_{2}\right.$ (bzac)(L5)]was more potent against $P$. aeruginosaand $S$. aureus with MIC value of $15.6 \mu \mathrm{g}$ and $\left[\mathrm{TiCl}_{2}\right.$ (bzac)(L4)]was more potent against $M$. luteus and $S$. epidermidiswith $15.6 \mu \mathrm{g}$ dose concentration. $\left[\mathrm{TiCl}_{2}(\mathrm{bzac})(\mathrm{L} 3)\right]$ and $\left[\mathrm{TiCl}_{2}(\mathrm{bzac})(\mathrm{L} 2)\right]$ were found to be more potent against $P$. aeruginosaand A. hydrophila, respectively, with $15.6 \mu \mathrm{g}$ concentrationof respected titanium (IV) complexes. $\left[\mathrm{TiCl}_{2}(\mathrm{bzac})(\mathrm{L} 1)\right]$ was more potent against $P$. aeruginosa with $62.5 \mu \mathrm{g}$ MICvalue compared to the other bacterial strains, respectively.

\section{Antifungal activity}

Haron et al. [8]synthesized phenyl fatty hydroxamic acids (PFHAs), shown in Fig. 3 by phenyl hydroxylaminolysis of canola or palm kernel oils using lipozyme TLIM as catalyst. Copper complexes as copper phenyl fatty hydroxamate (Cu-PFHs) were also prepared bystirring the phenyl fatty hydroxamic acids dissolved in hexane and copper(II) nitrate solution.The antifungal properties of phenyl fatty hydroxamic acids and its copper (II) complex, $\mathrm{Cu}-\mathrm{PFHs}$ were investigated against Candida parapsilosis, Candida albicans and Aspergillus fumigatus by the disc diffusion method. The results depicted that the antifungal activity of $\mathrm{Cu}-\mathrm{PFH}$ was higher as compared with phenyl fatty hydroxamic acids and also there is an increase in the activity of phenyl fatty hydroxamic acids and $\mathrm{Cu}$ PFHs with concentrations. 


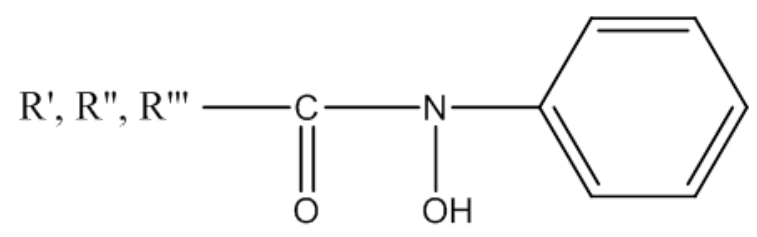

Fig. 3. Structure of phenyl fatty hydroxamic acid (PFHAs)

In a study carried out by Glensk et al. [9] the antimicrobial activity of natural cyclic hydroxamic acid 2,4dihydroxy-7-methoxy-2H-1,4-benzoxazin-3(4H)-one

(DIMBOA) was studied against yeasts-like reference strains of fungi using diskdiffusion method. It was observed that DIMBOA posesses growth inhibitory properties against Saccharomyces cerevisiae BCMM 3963 strain with an inhibition zone of $21.0 \pm 0.16 \mathrm{~mm}$.

Albalawi [10] synthesized novel hydroxamic acid compounds and further tested their antifungal activities against different fungal strains at concentration of $10 \mathrm{mg} / \mathrm{ml}$ in methanol. The fungal strains used were Candida krusei ATCC 6258, Candida parapsilosis ATCC 22019, Candida tropicalis and Candida albicans ATCC 2091. The diameters of zone of inhibition were variable from 12 and $22 \mathrm{~mm}$ depending on the strain tested. Out of the 4 hydroxamic acid derivatives, compound (S)-N-hydroxy-3-methyl-2(phenylsulfonamido)butanamide exhibited a very significant antifungal activity against Candida parapsilosis and Candida albicans.

3. Preparation of substituted hydroxamic acid and their antimicrobial activity

Antibacterial and antifungal activities of hydroxamic acids are connected with their ability to inhibit various enzymes, viz., matrix metalloproteinases [11] [12], urease [13] [14] or ribonucleotid reductase [15-17]. Agarwal et al. [18] has reported the synthesis and characterization of hydroxamic acids such as N-phenylbenzohydroxamic acids (PBHA), N-p-tolylbenzohydroxamic acid (PTBHA), N-otolylbenzohydroxamic acid (OTBHA), N-mchlorophenylbenzohydroxamic acid (MCBHA), N-pcarboxyphenylbenzohydroxamic acid (PCBHA), acetohydroxamic acid (AHA), benzohydroxamic acid (BHA) and salicylhydroxamic acid (SHA) as shown in Fig. 4 and the results of the undertaken antimicrobial evaluation. For the preparation of substituted hydroxamic acid $0.1 \mathrm{M}$ of hydroxylamine was taken in $25 \mathrm{ml}$ of benzene, distilled water and 0.02 Msodium bicarbonate in a $250 \mathrm{ml}$ Erlenmeyer's flask. Then 0.25 Mof benzoyl chloride was added to the solution, with constant shaking, till effervescence ceased. The water layer was kept alkaline to litmus by the gradual addition of sodium bicarbonate. The color of the reaction mixture turned from yellow to pink on addition of benzoyl chloride near completion of the reaction. The reaction took around 90 minutes for completion. Both mono- and di-substituted derivatives were formed. The solution was filtered, and the solid was washed with water. Di-substituted derivatives were removed using aqueous ammonia. The filtered ammonical solution, which was generally yellow or green, was added dropwise to slight excess of dilute sulphuric acid containing some crushed ice to yield the hydroxamic acid which was filtered, washed with water, and dried. The product was crystallized from 60:40 (v/v) ethyl alcohol-water mixture. For BHA direct benzoylation of hydroxylamine hydrochloride and for AHA the acylation of hydroxylamine hydrochloride was carried out.All the synthesized hydroxamic acids were tested for the in vitro growth inhibitory activity against the bacteria Enterobacter cloacae, Escherichia coli, Pseudomonas aeruginosa, Proteus vulgaris, Staphylococcus aureus (coagulase positive and coagulase negative) and the fungi Candida albicans by using the disc-diffusion method. It was concluded that out of six bacterial cultures tested, acetohydroxamic acid (AHA) and benzohydroxamic (BHA) acid exhibited wide spectrum of activity, as they were highly active against all cultures and the next one was salicylhydroxamic acid (SHA), as it was also active against all cultures. All of the other tested compounds exhibited moderate antimicrobial activity against all species of bacteria used in this study. The antifungal activity of the compounds was studied on Candida albicans. The results of fungicidal screening showed that among all the tested hydroxamic acids, BHA and AHA showed good activity while other compounds except PCBHA and SHA were effective moderately. The variation in the effectiveness of different compounds against different organisms depends either on the impermeability of the cells of the microbes or the difference in ribosome of microbial cells.


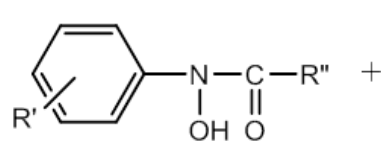

Mono-substituted Derivative



Di-substituted Derivative

Where, $\mathrm{R}^{\prime}=\mathrm{H},\left(p-\mathrm{CH}_{3}\right),\left(o-\mathrm{CH}_{3}\right),(m-\mathrm{Cl}),(p-\mathrm{COOH}),(p-\mathrm{OH})$; $\mathrm{R} "=\mathrm{C}_{6} \mathrm{H}_{5}, \mathrm{CH}_{3}, \mathrm{C}_{6} \mathrm{H}_{5}(o-\mathrm{OH})$

Fig. 4. Preparation of substituted hydroxamic acid 
Davis et al. [19] extensively studied the in vitro antimicrobial activity of some Cyclic Hydroxamic Acids against Enterobacter aerogenes, Serratia marcescens, Klebsiella pneumoniae, Pseudomonas aeruginosa, Escherichia coli, Lactobacillus casei, Lactobacillus plantarum, Leuconostoc dextranicum, and Streptococcus faecalis. The MIC of three cyclic hydroxamic acids, 3amino-3,4-dihydro-1-hydroxycarbostyril (ADHC), the 6chloro analog, and the 7-chloro analog, were 0.6, 0.6, and $0.2 \mathrm{ug} / \mathrm{mlrespectively}$ and those of the corresponding lactams, 3-amino-3,4-dihydrocarbostyril (ADC), the 6chloro analog, and the 7-chloro analog, were 60, 60, and 6 $\mathrm{ug} / \mathrm{ml}$, respectively. Structures of ADHC, ADC and their 7and 6-chloro analogs are depicted in Fig. 5.

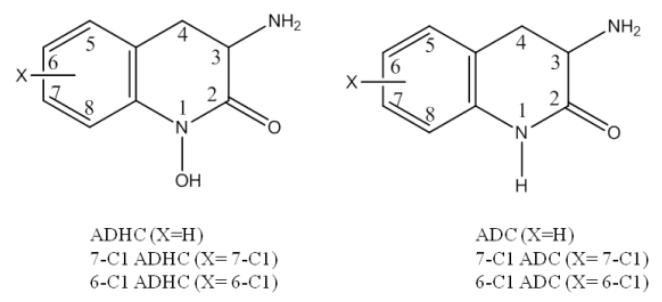

Fig. 5. Structures of ADHC, ADC and their 7- and 6-chloro $(\mathrm{X}=\mathrm{Cl})$ analogs

Table 1. Antibacterial activities of various hydroxamic acids and lactams (Modified from Davis et al.) ${ }^{[19]}$

\begin{tabular}{|l|l|l|l|l|l|l|}
\hline \multirow{2}{*}{$\begin{array}{c}\text { Microorganis } \\
\mathrm{m}\end{array}$} & \multicolumn{6}{|c|}{ MIC of various compounds $(\mu \mathrm{g} / \mathrm{ml})$} \\
\cline { 2 - 7 } & $\begin{array}{l}7-\mathrm{Cl} \\
\mathrm{ADHC}\end{array}$ & $\begin{array}{l}6-\mathrm{Cl} \\
\mathrm{ADHC}\end{array}$ & $\mathrm{ADHC}$ & $\begin{array}{l}7-\mathrm{Cl} \\
\mathrm{ADC}\end{array}$ & $\begin{array}{l}6-\mathrm{Cl} \\
\mathrm{ADC}\end{array}$ & $\mathrm{ADC}$ \\
\hline E. aerogenes & 0.06 & 0.06 & 0.2 & 6 & 60 & 60 \\
\hline S. marcescens & 0.02 & 0.06 & 0.2 & 6 & 60 & 60 \\
\hline K pneumoniae & 0.02 & 0.06 & 0.2 & 6 & 60 & 60 \\
\hline P. aeruginosa & 0.6 & 2 & 2 & 200 & $>200$ & $>200$ \\
\hline E. coli & 0.2 & 0.4 & 1 & 60 & $>200$ & $>200$ \\
\hline L. casei & 0.2 & 0.6 & 0.6 & 2 & 20 & 20 \\
\hline L. plantarum & 0.2 & 0.6 & 0.6 & 6 & 60 & 20 \\
\hline L. dextranicum & 6 & 10 & 20 & 20 & 60 & 200 \\
\hline S. faecalis & 6 & 20 & 20 & 200 & $>200$ & $>200$ \\
\hline Mean MIC & 0.2 & 0.6 & 0.6 & 6 & 60 & 60 \\
\hline
\end{tabular}

In addition, the cyclic hydroxamic acids but not the lactams inhibited the growth of Candida albicans at minimal inhibitory concentrations ranging from 20 to 200 $\mu \mathrm{g} / \mathrm{ml}$, at $\mathrm{pH}$ 7. Optimal antifungal activity of these compounds was observed at $\mathrm{pH} 7$ at which the 7-Cl $\mathrm{ADHC}$ and 6-Cl $\mathrm{ADHC}$ were 10 times more effective than $\mathrm{ADHC}$ as antimicrobial agents against $C$.albicans. Since the lactams were inactive as growth inhibitors of C.albicans even at concentrations up to $200, \mathrm{ug} / \mathrm{ml}$, these results suggested the importance of the hydroxamate group for antifungal activity. This study demonstrated that, the cyclic hydroxamic acids were much more effective antimicrobial agents than the structurally related lactams.

\section{Antiviral activity}

In order to study the effect of suberoylanilide hydroxamic acid (SAHA) on hepatitis $\mathrm{C}$ virus (HCV) replication, the HCV replicon cell OR6 was treated with SAHA. It was observed that $\mathrm{HCV}$ replication was significantly inhibited by SAHA at concentrations below 1 $\mu \mathrm{M}$ with no cellular toxicity. Tricostatin A (an HDAC inhibitor) also showed reduction of HCV replication. The liver specific microRNA-122, which is involved in HCV replication, was not affected by SAHA treatment. The results concluded that SAHA has suppressive effect on $\mathrm{HCV}$ replication through alterations of gene expression such as osteopontin (OPN) and apolipoprotein-Al (Apo-A1) in host cells. Treatment involving HDAC inhibitors may be considered as a novel therapeutic approach for diseases associated with HCV infection such as chronic hepatitis, liver cirrhosis, and HCC [20].

Like various other DNA viruses and retroviruses (e.g., herpes simplex virus, Kaposi's sarcoma-associated herpesvirus, simian virus 40, and murine leukemia virus), the Human Adenovirus (HAdV) DNA associates with cellular histones during specific stages of the virus life cycle. There is a need for an effective antiviral therapy for the treatment of these severe HAdV-induced diseases. Saha et al. [21] investigated the effect of pan-histone deacetylase (HDAC) inhibitor SAHA for the reduction of HAdV-5 gene expression and DNA replication, thereby decreasing virus yield from infected cells. It was found that SAHA also reduced gene expression from more virulent and clinically relevant serotypes, including HAdV-4 and HAdV-7. In addition to SAHA, several other HDAC inhibitors (e.g., trichostatin A, apicidin, and panobinostat) also affected HAdV gene expression.

Antibiotic resistance among Gram-positive bacteria (Staphylococci, Enterococci, and Streptococci) is becoming a major health concern. Therefore, there is an urgent need to discover antibiotics with new modes of action [22]. Peptide deformylase (PDF), a metalloenzyme was considered as an important target in antibacterial drug discovery. Peptide deformilase (PDF) is believed to be an essential enzyme in both Gram-positive and Gram-negative bacteria [23]. Subsequent studies have shown that the instability is due to the oxidation of a ferrous ion at the enzyme's active site. In Eubacteria, protein synthesis is initiated with Nformylmethionine. In most cases, the newly synthesized polypeptide is converted to mature protein through the sequential removal of the $\mathrm{N}$-formyl group and methionine by peptide deformylase and methionine amino peptidase (MAP) respectively as shown in Fig. 6. In some cases, the formyl group is removed but the initiator methionine is retained. 


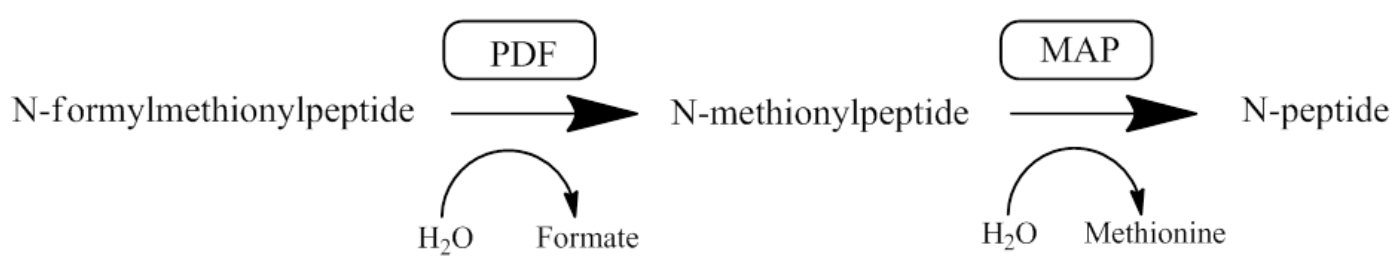

Fig. 6. Reaction catalyzed by PDF and MAP metalloproteinase enzymes (MMPs) [14], [22], [24].

In Escherichia coli, PDF 1 is encoded by the def gene, homologues of which are present in all bacterial genomes sequenced to date. In those cases that have been studied, the PDF enzymes share many common properties. In the past decade, there has been a dramatic increase in the number of reports of pathogenic bacteria that are resistant to currently available antibacterials. This is partially due to the fact that all the available antibacterials belong to a limited number of chemical classes, which target a similarly limited number of molecular targets of the bacteria. To develop new antibacterial drugs that work by inhibiting novel targets, many new technologies are being used and several criteria for selecting new targets have been proposed. Bacterial PDF appears to satisfy several of these criteria: it is essential to bacterial growth and has no mammalian counterpart. Since there are no clinically used antibacterial agents that target PDF, bacteria already resistant to existing antibiotics should remain susceptible to PDF inhibitors. PDF also is a very attractive target for drug design since it belongs to the family of metallohydrolases, one of the best studied enzyme classes, and there is a rich literature on how to search for and design inhibitors of enzymes of this type [23].Since PDF is a metallohydrolase, compound containing a metal ion, the chelating groups are potential inhibitors of the enzyme. Rather than screen empirically for inhibitors of PDF, most of the researchers employ a strategy relying on the fact that the target is a metalloenzyme.

Actinonin as shown in Fig. 7 (A) is a known hydroxamate containing inhibitor of several metallo hydrolases and earlier described as an aminopeptidase inhibitor. The hydroxamate containing compounds are very potent inhibitors of metalloenzymes, especially Matrix
Although several different chelating groups have been reported in the literature, hydroxamate remains the preferred group. The hydroxamate group of actinonin evidently acts as the chelating group to bind the metal ion of the enzyme. Previously, the hydroxamate group of actinonin was shown to be essential for maintaining the antibacterial activity [25]. In addition, modifications of the proline group at P3' could be made without loss of their activity, provided that the pseudopeptide backbone is retained. Indeed, it has been determined that [4-(N-hydroxyamino $)-2(R)-$ isobutylsuccinyl]- $L$-tert-butylglycine-N-ethylamide, a hydroxamate-containing pseudopeptide without proline at the P3' site, as shown in Fig. 7 (B) [26] is still a potent PDF inhibitor $\left(\mathrm{IC}_{50}=5 \mathrm{nM}\right)$. Taken together, these data suggest that the hydroxamate, and not proline at the P3' site, is primarily responsible for the potent PDF inhibitory activity of actinonin $\left(\mathrm{IC}_{50}=0.006 \mu \mathrm{M}\right)$. It is not clear why hydroxamate is the preferred metal-binding group, but polar interactions between the hydroxamate and enzyme residues in the vicinity of the metal binding site could contribute to the high affinity. Gao et al. [27] have identified highly potent inhibitors of peptide deformylase. Antibacterial activity suggested that the compound which they classified as vanillin hydroxamic acid was highly active against bacterium and especially fungus. The binding mode of this compound also indicated that it may serve as a new class of PDF inhibitors for development of antibacterial compounds. The orally bioavailable N-formyl hydroxylamine BB-3497 as shown in Fig. 7 (C) has given momentum to the validity of PDF as a novel antibacterial target, with an $\mathrm{IC}_{50}$ of $7 \mathrm{nM}$ [28].

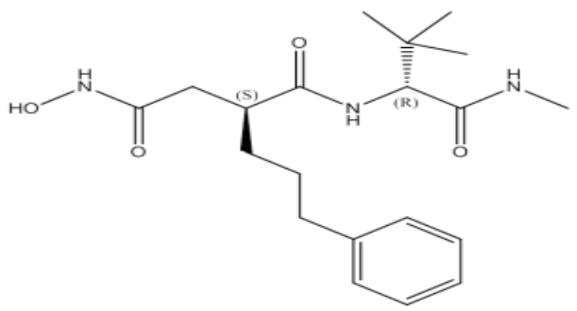

(B) 4-(N-hydroxyamino)-2(R)isobutylsuccinyl]-L-tert-butylglycine-Nethylamide



Fig. 7. Hydroxamate-containing inhibitors of PDF 
Alkiewicz et al. [29]reported that salicylhydroxamic acid completely inhibits growth of some pathogenic fungi (Trichophyton schoenleinii, T. gypseum, T. rubrum, T. violaceum) in vitro at a concentration of $0.025-0.1 \mathrm{mg} / \mathrm{ml}$.). Similar effectswere observed byuse of benzohydroxamic acid and 2-hydroxy-3-naphthydroxamic acid.

\section{B. Anti inflammatory activity}

Flynn et al. [30] have identified a new dual cyclooxygenase and 5 lipooxygenase inhibitor (COX/5-LO inhibitor), a pyrazole-containing hydroxamic acid, tepoxalin as shown in Fig. 8 that has received extensive biochemical and clinical evaluation as an anti-inflammatory agent.

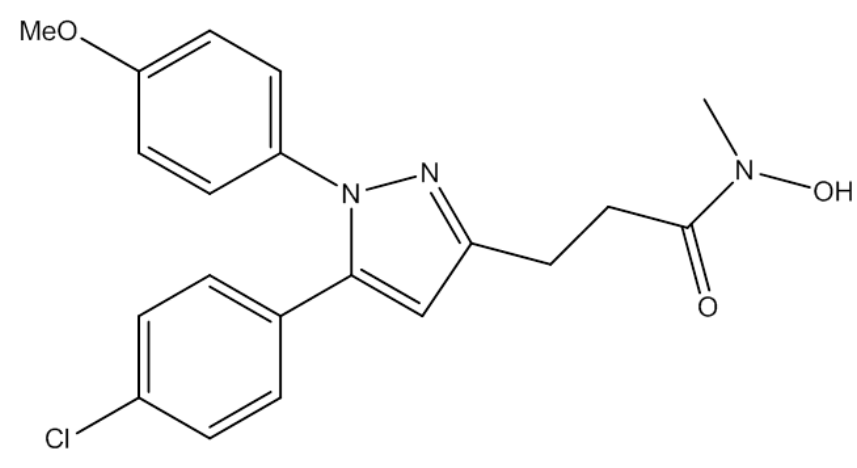

Fig. 8. Tepoxalin

The cyclooxygenase (COX) enzyme is responsible for the conversion of arachidonic acid into prostaglandins (PG's). Elevations in both prostaglandins $\left(\mathrm{PGE}_{2} / \mathrm{PGF}_{2} \alpha\right)$ and leukotrienes $\left(\mathrm{LTB}_{4}\right)$ have been reported in numerous inflammatory processes. It is now well established that COX exists as two isoforms in mammals, a constitutive form (COX-1), and an inducible form (COX-2). It is currently hypothesized that the undesirable side-effects of nonsteroidal anti-inflammatory drugs (NSAIDs) are due to COX-1 inhibition, whereas the beneficial effects, such as reduction of swelling and analgesia, are related to COX-2 inhibition. A promising alternative to avoid the adverse reactions of NSAIDs could be the development of drugs that inhibit both COX and 5-Lipoxygenase (5-LO) leading to compounds with improved efficacy [31]. These findings stimulated several groups to design new potent molecules incorporating a hydroxamate moiety into the wide range of existing NSAID pharmacophores. To this end, Flynn and coworkers have prepared various hydroxamic acid derivatives of known NSAID compounds as meclofenamic acid, indometacin, sulindac and ibuprofen. These compounds appear to be balanced dual inhibitors of COX and 5-LO. Many of these new dual COX/5-LO inhibitors also exhibit potent topical anti-inflammatory activity in the arachidonic acid-induced murine ear edema model. The most potent compound in this series, HP 977 shown in Fig. 9, showed a good profile of in vitro and in vivo activities [30].

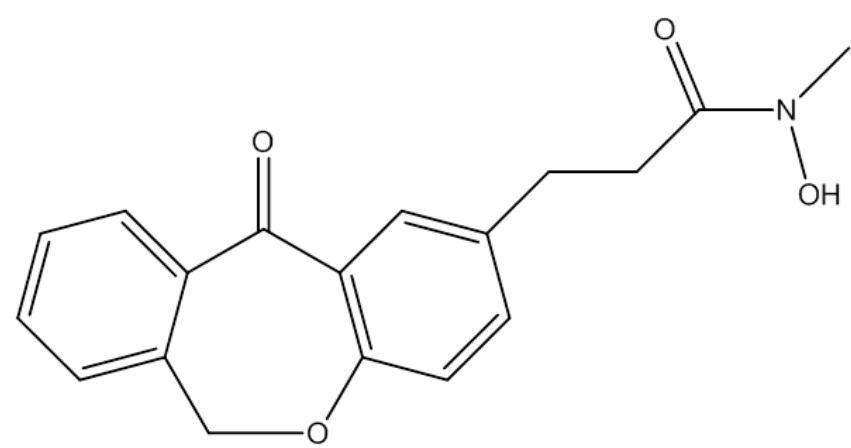

Fig. 9. HP 977

Lipoxygenase plays an essential role in the biosynthesis of the leukotrienes. Leukotrienes, as LO metabolites of arachidonic acid (AA), have been implicated as mediators in the pathophysiology of inflammatory diseases, host defense reactions and to play important role in the propagation of the diseases states, exacerbating the local events and ultimately leading to tissue damage. Simple stable molecules containing the hydroxamic acid functionality have been shown to inhibit 5-lipoxygenase. In fact, several hydroxamates are orally active inhibitors of the enzyme as determined by their ability to block the biosynthesis of leukotriene in vivo. Similar results have been given by [32], establishing the inhibitory utility of simple hydroxamates and omega-phenylalkyl and omeganaphthylalkyl hydroxamic acids. The compounds were tested in vitro on soybean lipoxygenase inhibition, and reported medium anti-inflammatory activity and potent inhibition of soybean lipoxygenase as a result of their physicochemical features.

In an attempt to regulate a variety of human conditions, including chronic inflammatory diseases, Vorinostat and other inhibitors of different histone deacetylase (HDAC) enzymes are being found. The dose-dependent pro- and antiinflammatory efficacy of two known inhibitors of multiple HDACs, Vorinostat and BML281, were investigated in human macrophages and in a rat model of collagen-induced arthritis by monitoring effects on disease progression, histopathology and immunohistochemistry. Both HDAC inhibitors differentially modulated lipopolysaccharide (LPS)-induced cytokine release from human macrophages, suppressing release of some inflammatory mediators (IL12p40, IL6) at low concentrations $(<3 \mu \mathrm{M})$ but amplifying production of others (TNF, IL1 $\beta$ ) at higher concentrations $(>3 \mu \mathrm{M})$. This trend translated in vivo to rat arthritis, with anti-inflammatory activity inversely correlating with dose. Both compounds were efficacious only at a low dose $(1 \mathrm{mg} / \mathrm{kg} /$ day $)$, whereas a higher dose $(5$ $\mathrm{mg} / \mathrm{kg} /$ day) showed no positive effects on reducing pathology, even showing signs of exacerbating disease. The findings support new investigations into repurposing HDAC inhibitors for anti-inflammatory therapeutic applications. However, HDAC inhibitors should be reinvestigated at lower, rather than higher, doses for enhanced efficacy in chronic diseases that require long-term treatment, with careful management of efficacy and long-term safety [33]. Effects of suberoylanilide hydroxamic acid (SAHA) on the 
viability and apoptosis of rheumatoid arthritis of fibroblastlike synoviocytes (rheumatoid arthritis (RA) fibroblast-like synoviocytes (FLS)) were studied by [34]. They treated the FLS obtained from RA patients with SAHA. SAHA-treated FLS showed a significant increase in the percentage of apoptosis and the expression and activity of caspase- 3 and higher intracellular ROS levels [34].

\section{Anticancer activity}

Researchers have put considerable efforts on attempting to exploit the characteristics of a new class of antitumour agents - histone deacetylase (HDAC) inhibitors for anticancer therapies. HDACs are involved in different cellular pathways and functions. HDAC inhibitors induce cancer cell cycle arrest, differentiation and cell death, reduce angiogenesis and modulate immune response. HDAC inhibitors seem to be promising anti-cancer drugs particularly in the combination with other anti-cancer drugs and/or radiotherapy. HDAC inhibitors vorinostat, romidepsin and belinostat have been approved for some Tcell lymphoma and panobinostat for multiple myeloma [35].

Hydroxamic acids have been found to react with both proteins and nucleic acids [36]. The reactivity of hydroxamic acids towards sulfhydryl groups of proteins has been suggested to be the reason for their inhibitory effect on various enzymes. The protease papain, for instance, with a single free cysteine residue located at the active site was irreversibly inhibited by DIMBOA (2,4-dihydroxy-7methoxy-1,4-benzoxazin-3-one). Friebe et al. [37]showed an inhibitory effect of DIBOA (2,4-dihydroxy-1,4-benzoxazin3 -one) and DIMBOA on plasma membrane $\mathrm{H}^{+}$-ATPase from roots of Avena sativa and Avena fatua. This inhibition may also be due to the reactivity of hydroxamic acids towards sulfhydryl groups since at least one exposed cysteine residue at the active site is of importance for maintenance of enzyme conformation. In addition, DIMBOA was shown to have an inhibitory effect on the electron transport in isolated mitochondria and chloroplasts of maize [38]. Also, [36] showed both DIBOA and DIMBOA to be mutagenic in a test with Salmonella typhimurium. The reactivity of hydroxamic acids offers an explanation to the various biological effects observed.

Suberoylanilide hydroxamic acid (SAHA), shown in Fig. 10 is a synthetic hydroxamic acid derivative with antineoplastic activity. It is a second generation polar-planar compound which binds to the catalytic domain of the histone deacetylases (HDACs). This allows the hydroxamic moiety to chelate zinc ion located in the catalytic pockets of HDAC, thereby inhibiting deacetylation and leading to an accumulation of both hyperacetylated histones and transcription factors. Hyperacetylation of histone proteins results in the upregulation of the cyclin-dependant kinase p21, followed by G1 arrest. Hyperacetylation of non-histone proteins such as tumor suppressor p53, alpha tubulin, and heat-shock protein 90 produces additional anti-proliferative effects. This agent also induces apoptosis and sensitizes tumor cells to cell death processes. Vorinostat is a small molecular weight (less than 300) linear hydroxamic acid compound that inhibits HDAC activity thereby inducing the accumulation of acetylated histones as well as nonhistone proteins, blocks the proliferation of cultured cells, and inhibits tumour growth in a variety of animal models. Vorinostat is a broad inhibitor of HDAC activity on cancer cell lines [39], [40]. Crystallographic studies have revealed that vorinostat inhibits HDAC activity by binding in the active site of the enzyme [41]. As shown in Fig. 11, the hydroxamic end of the molecule binding to the zinc atom in the HDAC catalytic site, with the phenyl ring of vorinostat projecting out of the catalytic pocket on to the surface of HDAC.<smiles>O=C(CCCCCCC(=O)Nc1ccccc1)NO</smiles>

Fig. 10. N-hydroxy-N'-phenyl-octanediamide/ Vorinostat or suberoylanilide hydroxamic acid (SAHA)

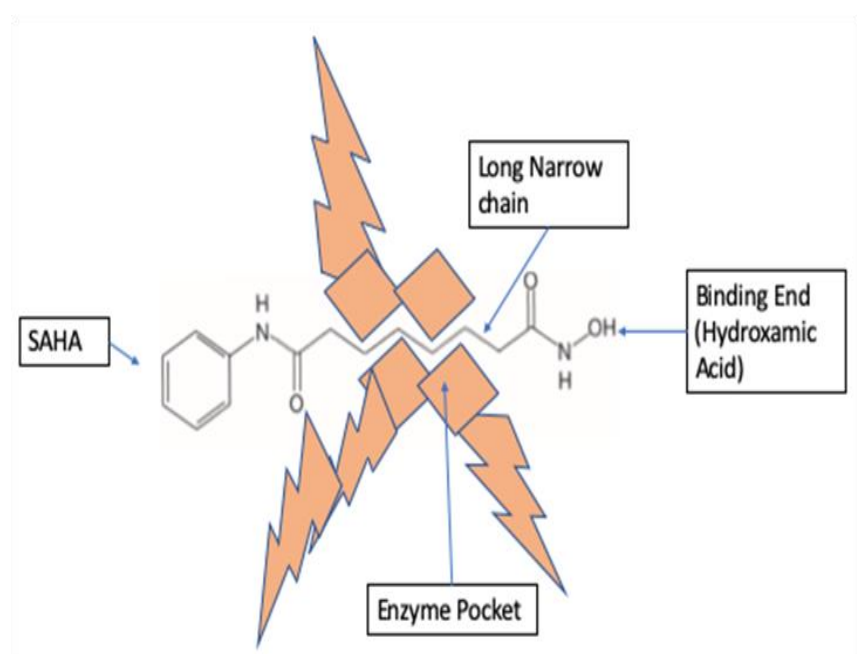

Fig. 11. Vorinostat inhibits HDAC activity by binding to the pocket of the catalytic site. The hydroxamic acid moiety of vorinostat binds to a zinc atom (pink), allowing the rest of the molecule to lie along the surface of the HDLP protein ${ }^{[42]}$

This small-molecule drug inhibits the activity of a group of enzymes called histone deacetylases (HDACs), which remove small chemical groups called acetyl groups from many different proteins, including proteins that regulate gene expression. By altering the acetylation of these proteins, HDAC inhibitors can induce tumor cell differentiation, cell cycle arrest, and apoptosis. Acetyl groups on the lysine tails of DNA associated histone proteins interact with the phosphate backbone of DNA and in doing so allow access of transcription factors to DNA. Deacetylation of histone proteins by HDACs results in compacted and therefore, inaccessible chromatin. In the presence of HDAC-inhibitors, chromatin remains in an opened configuration, allowing transcription factors to reach DNA promoters and facilitate transcription of tumor suppressor genes that check the growth of cancer cells. HDAC-inhibitors including SAHA have a wide variety of functions important for treating cancers including induction of apoptosis or differentiation, cell cycle arrest, and 
inhibition of angiogenesis [40]. Major known activities of SAHA are shown in Fig. 12having a close relationship with the concept of modification cassette and localized binary switches, where the adjacent amino acid sites are modified within specific neighboring histone tails acting as information units for different signals [43]. The HDACs determine the pattern of acetylation of various lysine's in the tail part of the histones resulting in a generation of a code recognizable by non-histones proteins forming complexes involved in the regulation of gene expression including the information whether the histone modification was initiated as a stand-alone, sequentially or in a combination of other metabolic processes in the cell [43], [44], [45]. SAHA was able to induce apoptosis and cell cycle arrest in cutaneous $\mathrm{T}$ cell lymphoma (CTCL) cell lines and malignant cells from Sézary Syndrome patients' blood [46]. Furthermore, there was nuclear expression of the transcription factor, $\mathrm{p}-\mathrm{Stat}-3$ at baseline and it became cytoplasmic in skin lesions from patients with clinical responses to SAHA [39].



Fig. 12. Proposed mechanism of action of SAHA

Studies with SAHA have primarily been performed with hematopoietic tumor cells. Huang and Pardee [47] extended these studies with SAHA to human breast cancer cell lines and concluded it as better therapeutic agents for breast cancer treatment. Through their studies they demonstrated that SAHA induces cytotoxic effects on human breast cancer cells with significantly lower toxicity on normal cells, including normal breast epithelial cells and fibroblasts. Moreover, SAHA induces cell cycle arrest and eventual apoptosis in human breast cancer cells, possibly by modulating cell cycle and apoptosis regulatory proteins, such as the retinoblastoma protein (pRb) and cyclin dependent kinase (CDK) inhibitors p21 and p27. Furthermore, SAHA induces several genes associated with differentiation and/or growth inhibition, such as gelsolin, isopentenyl- diphosphate-delta isomerase (IDI1) and 1, 25 dihydroxyvitamin D-3 upregulated protein 1 (VDUP1), which may contribute to SAHA-mediated pro-differentiating and antiproliferative effects.

Wang and Lee [4] using proteomics tools concluded that SAHA is a potential inhibitor of cervical cancer. In their studies five proteins were found to be downregulated by SAHA - Phosphoglycerate mutase 1 (PGAM1), Tubulinspecific chaperone A (TBCA), Heat-shock protein beta-1 (HSPB1), Aldose reductase (ALDR) and Eukaryotic translation initiation factor 5A-1 (IF5A1). The functions of the changed proteins were correlated to apoptosis and/or antiproliferation cellular processes. Furthermore, significant downregulation of PGAM1 after treatment with SAHA was confirmed in two cervical cancer cell lines (HeLa and CaSki) by western blot analysis. These changed proteins may provide some clues to a better understanding of the molecular mechanisms underlying SAHA-induced apoptosis in cervical cancer. Similarly, [48] also utilized the proteomic approach to evaluate if SAHA had an activity against liver cancer. A variety of differentially expressed proteins were identified in human HepG2 cancer cells before and after treatment with SAHA. Six proteins were identified and displayed significant expression changes at earlier time points upon SAHA treatment. Together, at both the mRNA and protein levels, SAHA suppressed the expression of reticulocalbin 1 precursor (RCN1), annexin A3 (ANXA3) 
and heat shock $27 \mathrm{kDa}$ protein 1 (HSP27), while increasing the expression of aldose reductase (AR), triosephosphate isomerase 1 (TPI) and manganese superoxide dismutase (SOD2). This study enabled a better understanding of the molecular mechanisms underlying SAHA mediated antitumor effects at the protein level and proved SAHA as a remarkable inhibitor of proliferating HepG2 liver cancer cells.

Apoptosis is an important mechanism by which anticancer agents induce cancer cell death. Both sulindac [2[6-fluoro-2-methyl-3-[(4-methylsulfinylphenyl)

methylidene]inden-1-yl]-acetic acid], shown in Fig. 13 and SAHA are promising anticancer therapeutics with independent pathways of action that are currently in clinical trials. Sulindac acts through inhibition of Cox-2, whereas SAHA seems to induce the expression of specific target genes to stimulate growth arrest, differentiation, or apoptosis of malignant cells. Seo et al. [49] demonstrated that a combination of sulindac and SAHA at the suboptimal dose of $250 \mu \mathrm{M}$, sulindac significantly enhances SAHA-induced growth suppression and apoptosis of human non-small cell lung cancer cells, primarily via enhanced collapse of the mitochondrial membrane potential, release of cytochrome $c$, and caspase activation than either agent alone. Their findings indicated that a combination of these two agents can be used to kill lung cancer cells more effectively with minimal side effects.

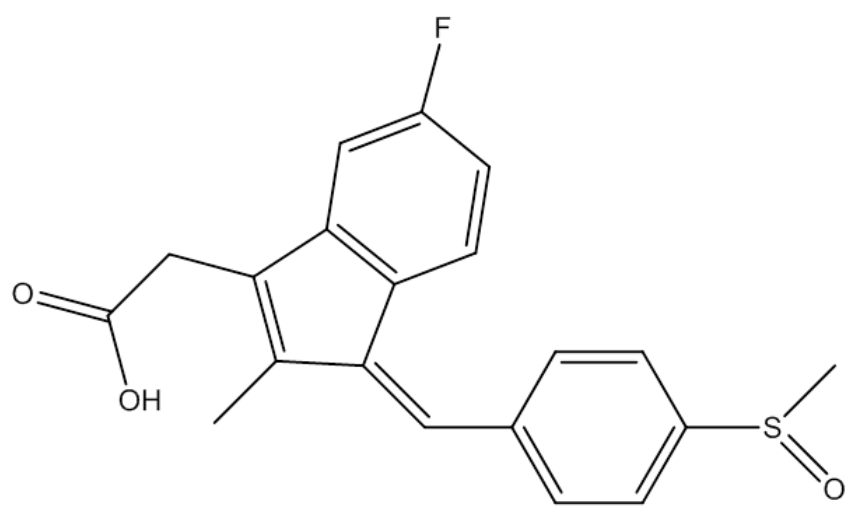

Fig. 13. Sulindac

A novel series of hybrids were designed and synthesized from $\beta$-carboline and hydroxamic acid. These compounds have not only shown significant antiproliferation activity against four human colorectal cancer (CRC) cell lines, but also showed histone deacetylase inhibitory effects in vitro [50]. A new series of acridine hydroxamic acid derivatives targeting HDAC were designed and synthesized. All these compounds not only displayed potent antiproliferative activities against U937 cells in vitro but also showed HDAC1/6 inhibition activity [51].

There have been various articles concerning the biochemistry of anticancer activity of naturally occurring and synthetic class of organic compounds containing the hydroxamic acid functional group (-CONHOH). Hydroxyurea containing that group is a well-known anticancer drug as reported by [52]. It inhibits the DNA synthesis by impairing the activity of enzyme ribonucleotide reductase. Though it is clinically used as anticancer agent. Elford et al. [53] found that it perturbs the hematological parameters and depresses the bone marrow. Recently [54] has reported that cholorohydroxamic acid possesses antitumor properties and inhibits the growth of Ehrlich ascites carcinoma (EAC) cells by imparing DNA and protein synthesis without altering the hematological parameters. Similarly, [53] has reported significant antitumor activity in benzohydroxamic acid.

\section{Antimalarial activity}

Malaria still remains one of the most important parasitic disease of the developing world although it is known to humankind since ancient times in different forms. It is caused by Plasmodium malarial parasite and kills approximately 1-3 million people and causes disease in 300 - 500 million people annually. The malaria parasite is a Plasmodium protozoan species, which evolved with time differentiating into four distinct species; P. falciparum, $P$. vivex, $P$. malarae and $P$. ovale, specific to man. Out of different strains causing this disease $P$. falciparum is a deadly one and is very common in tropical Africa, South America and South East Asia including the Indian subcontinent and responsible for more than 95\% of malaria related deaths. Malaria control efforts include attempts to develop effective vaccine, eradicate mosquito vectors, and develop new drugs. The increasing burden of malaria partially due to parasite drug resistance demands continued and sustained improvement in antimalarial medicines through focused research and development. Therefore, there is an urgent need for new antimalarial agents that act on different parasite targets, as they have the potential to combat resistance. The histone deacetylases (HDACs) of $P$. falciparum (PfHDACs) may be promising targets for new classes of antimalarial drugs [55] [56]. Inhibitors of histone deacetylases (HDAC-Is) have been shown effective in controlling the development of many parasites, such as the plasmodium of malaria. Hydroxamate-based histone deacetylase inhibitors, having an amide-lactam moiety are more powerful compared to linear counterpart. Among the protozoa studied, $P$. falciparum was the most sensitive to this class of drugs. This class of HDAC inhibitor could be a new tool against malaria and is therefore a useful hit compound for further medicinal chemistry optimization [57]. A series of 24 peptoid-based HDACi was evaluated for anti-plasmodial activity and cytotoxicity against mammalian cells. All compounds displayed potent submicromolar activity against drug sensitive and drug resistant asexual blood stage $P$. falciparum parasites with IC50 values ranging from 4-158 $\mathrm{nM}$ and 1-118 $\mathrm{nM}$. Key compounds were further tested for activity against different $P$. falciparum gametocyte stages as well as against $P$. berghei exoerythrocytic forms. While only moderate activity was found against gametocytes, several compounds revealed submicromolar activity against $\mathrm{P}$. berghei exo-erythrocytic forms [58]. Falcilysin (FLN) is a malarial metalloprotease essential for parasite development in the human host. In a study the optimization of a piperazine-based hydroxamic acid scaffold to develop the first reported inhibitors of FLN was done. Inhibitors were tested against cultured parasites, 
and parasiticidal activity correlated with potency against FLN. This suggests these compounds kill P. falciparum by blocking FLN. These compounds represent an important step towards validating FLN as a therapeutic target and towards the development of chemical tools to investigate the function of this protease [59]. Some benzoyl hydroxamates have shown very good in vitro antimalarial activity. Very recently glycosyl hydroxamates were also found to possess antimalarial activities. The mode of action of these hydroxamates was established as chelation of iron and inhibition of the enzyme peptide deformylase [60]. Among the glycosyl hydroxamates compound possessing long alkyl chain were found to be most active [61].

Drug therapy is the mainstay of antimalarial therapy, yet current drugs are threatened by the development of resistance, so it's imperative to develop new antimalarials with great potency against both drug-susceptible and drugresistant malaria. Triazoles, bearing a five-membered heterocyclic ring with three nitrogen atoms, exhibit promising in vitro antiplasmodial and in vivo antimalarial activities. Moreover, several triazole-based drugs have already used in clinics for the treatment of various diseases, demonstrating the excellent pharmaceutical profiles. Therefore, triazole derivatives have the potential for clinical deployment in the control and eradication of malaria. Triazole derivatives such as triazole-quinoline/quinolone, triazole-coumarin/flavones/chalcone, triazole-indole/isatin, triazole- $\beta$-lactam, triazole-sugar, triazole-hydroxamic acid, triazole-pyrimidine, triazole-azole/azoline and triazolethiolactone/amidine/urea hybrids are considered as potential antimalarials [62]. Most hydroxamates that are used as antimalarial drugs acts as a chelator. The biological action has been attributed to chelation of internal iron pools and in turn to interference with the supply of iron to different components possibly to ribonucleotide reductase.

Desferrioxamine B shown in Fig. 14 is a polyhydroxamate iron chelator that is useful for reducing iron concentration in human blood plasma. It has the desirable property of high affinity for ferric ion $(\mathrm{Ka}=1031)$ coupled with a very low affinity for calcium $(\mathrm{Ka}=102)$ [60].

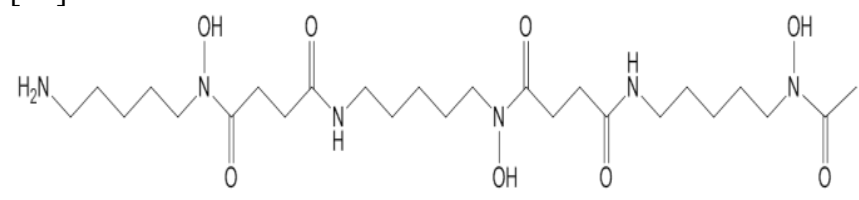

Fig. 14. Desferrioxamine B

\section{E. Antitubercular activity}

Rao et al. [63] evaluated the potential of the histone deacetylase inhibitors (HDIs) valproic acid (VPA) and (SAHA) in order to enhance the effects of first-line anti-TB drugs against intracellular Mycobacterium tuberculosis. The cultures of M. tuberculosis H37Rv were exposed to VPA or SAHA over a period of 6 days, in the presence or absence of isoniazid (INH) and rifampicin (RIF). Infected macrophages were selected for studying the efficiency of VPA and SAHA against intracellular M. tuberculosis with and without INH or RIF. Bactericidal activity was determined by counting mycobacterial colony-forming units (CFU). It was found that VPA treatment exhibited superior bactericidal activity as compared to SAHA (2-log CFU reduction), while both HDIs moderately improved the activity of RIF against extracellular M. tuberculosis. Also the bactericidal effect of VPA was greater than that of SAHA (1-log CFU reduction) and equalled that of INH (1.5-log CFU reduction) against intracellular M. tuberculosis. It was concluded that INH/RIF and VPA/SAHA combination treatment inhibited intracellular M. tuberculosis survival in a shorter time span than monotherapy (3 days vs. 6 days).

\section{F. Antioxidant activity}

Antioxidant compounds in food play an important role as a health- protecting factor. Scientific evidence suggests that antioxidants reduce the risk for chronic diseases including cancer and heart disease. Primary sources of naturally occurring antioxidants are whole grains, fruits and vegetables. Plant sourced food antioxidants like vitamin $\mathrm{C}$, vitamin E, carotenes, phenolic acids, phytate and phytoestrogens have been recognized as having the potential to reduce disease risk. Most of the antioxidant compounds in a typical diet are derived from plant sources and belong to various classes of compounds with a wide variety of physical and chemical properties. Some compounds, such as gallates, have strong antioxidant activity, while others, such as the mono-phenols are weak antioxidants. The main characteristic of an antioxidant is its ability to trap free radicals. Highly reactive free radicals and oxygen species are present in biological systems from a wide variety of sources. These free radicals may oxidize nucleic acids, proteins, lipids or DNA and can initiate degenerative disease [64]. A growing body of evidence suggests that endo- and exogenous reactive oxygen species (ROS) may have an important role in the expansion and progression of tumors. Reactive oxygen species (ROS), along with reactive nitrogen species (RNS), may play an important role in the pathogenesis and progressing of many diseases. The cumulative production of ROS/RNS through either endogenous or exogenous insults is termed oxidative stress and is responsible for or contributes to many pathological conditions [65]. For example, inflammatory tissue injuries are mediated by reactive oxygen metabolites from phagocytic leukocytes (e.g., neutrophils, monocytes, macrophages, and eosinophils) that invade the tissue. These reactive radicals and oxidants may injure cells and tissue directly via oxidative degradation of essential cellular components or indirectly by altering the protease/antiprotease balance that normally exists within the intestinal tissue [66]. The research has shown that the various types of cancer examined to date exhibit an imbalance in their antioxidant mechanisms. In the near future new insights in cancer therapies, based on modulation of cellular redox status, may lead the way to additional tools against carcinogenesis induced by ROS [67]. Oxidative stress is an imbalanced state of oxidants and antioxidants in organism, initiated by endogenous ROS, such as superoxide anion and hydrogen peroxide. These moderately strong oxidants can, in reaction with substances such as nitric oxide (NO) or transition metals, be converted into species with 
pronounced reactivity such as peroxynitrite and hydroxyl radicals. Those are molecules capable of attacking sensitive cellular targets like lipids, proteins and nucleic acids causing their inhibition and accelerated degradation. One of the ROS-mediated causes of cancer is gene mutations (modification of pyridine and purine bases) and posttranslational modifications leading to disruption of cellular processes [68]. Therefore, in the last decade hydroxamic acid compounds have received increased attention from medical researchers for their antioxidant activities. Koncic et al. [69] have investigated radical scavenging, metal chelating and antioxidant activities of several Cycloalkyl- $N$ arylhydroxamic acids shown in Fig. 15 viz. $N$-(4chlorophenyl)- $N$-hydroxycyclopropanecarboxamide, $\quad N$-(4chlorophenyl)-3-cyclopentyl- $N$-hydroxypropanamide, $N$-(4chlorophenyl)- $N$-hydroxycyclo hexanecarboxamide, $N$-(4chlorophenyl)-2-cyclohexyl- $N$-hydroxyacetamide, $N$-(4-

chlorophenyl)-3-cyclohexyl- $N$-hydroxypropanamide, $\quad N$ phenyl-2-furohydroxamic acid by scavenging effect on the 2,2-diphenyl-1-picrylhydrazyl (DPPH) free radical, metal chelation effect in the $\mathrm{Fe}^{2+}$-ferrozin test system, as well as by $\beta$-carotene-linoleic acid assay.

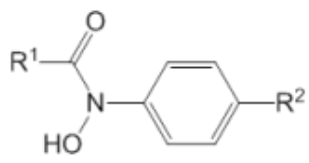

(1)

Fig. 15. Cycloalkyl- $N$-arylhydroxamic acids (1-6)

Hydroxamic acids investigated in this study were good radical scavengers, showed excellent chelation of ferrous ions. They also demonstrated pronounced antioxidant activities in the prevention of heat-induced oxidation of linoleic acid. The results of this study imply that investigated hydroxamic acids may also have potential in treatment of Sickel Cell Disease (SCD) due to their antioxidant, antiradical and chelating properties.

In order to improve the antioxidant and antimicrobial abilities, hydroxamated degraded polysaccharides from Enteromorpha prolifera (HCDPE) were prepared from the corresponding carboxymethylated degraded polysaccharides (CDPE). The in vitro antioxidant activity of HCDPE was evaluated by determining the radical (1,1-diphenyl-2picrylhydrazyl (DPPH), hydroxyl and superoxide anion radicals) scavenging abilities and total antioxidant activity. It was found that DPPH radical scavenging ability and total antioxidant activity of HCDPE were significantly improved compared to those of CDPE. The inhibitory effects of polysaccharides against the five bacterial strains (Bacillus subtilis, Staphylococcus aureus, Escherichia coli, Pseudomonas aeruginosa, Salmonella spp.) were also evaluated by bacterial inhibition zone and minimum inhibitory concentration (MIC) assays. The results indicated that CDPE and HCDPE possess marked antimicrobial ability, while such an effect was not observed for the crude polysaccharides (PE) and the degraded polysaccharides (DPE) [70]. In order to improve the bioactivity of the polysaccharide from Sargassum fusiforme (PSF), the degraded polysaccharide (DPSF) was modified by carboxymethylation, yielding carboxymethylated degraded polysaccharides (CDPSF), which were further modified to generate hydroxamated derivatives (HCDPSF). The in vitro antioxidant activity of CDPSF and HCDPSF was evaluated by determining the radical scavenging ability and total antioxidant activity. The results indicated that the antioxidant activity of CDPSF and HCDPSF was significantly improved when compared to those of DPSF. Antimicrobial assays indicated that both CDPSF and HCDPSF possessed a marked antimicrobial ability, while DPSF did not exhibit such effects under the same conditions. Such polysaccharide derivatives have potentials in the pharmaceutical and food industries [71].

\section{CONCLUSION}

Molecular hybridization has become a new promising way to treat multifactorial diseases with a single compound that acts on multiple targets. The combination of several functional pharmacophore groups in one molecule can lead to a stronger therapeutic effect due to the ability to bind to several targets and possible synergistic interactions. Hybrid compounds of hydroxamic acids are a potential basis for the development of effective drugs used in the complex treatment of oncological, infectious and neurological diseases. Recent developments in drug discovery have highlighted the numerous biological and pharmacological properties that the hydroxamic acid function may possess, leading to therapeutic applications. Due to the ability of hydroxamic acids to form complexes with various metal ions, in particular iron, zinc, magnesium and calcium, they possess a number of unique biological and pharmacological properties. Antibacterial, antifungal, antitumor and antiinflammatory activities of hydroxamic acids are due to their ability to inhibit various enzymes, such as matrix metalloproteinases, urease and peroxidase. Anticancer properties of some aliphatic and aromatic hydroxamic acids have also been studied. Suberoylanilide hydroxamic acid (SAHA) has been proved as potent drug for cancer therapy. Antioxidant properties of hydroxamic acids have also been observed. These properties can be divided into two groups. 
The first concerns the ability of these hydroxamic acid derivatives to scavenge metals (particularly iron), which leads to antioxidant, antimicrobial and metal detoxification activities. The latter is largely used to treat iron overload in patients. The second group of activities is related to their ability to inhibit metallo-enzymes, which gives them a wide range of pharmacological effects: antimicrobial, antiinflammatory and antitumor. Realizing the full biomedical potential of hydroxamic acid further efforts are needed to synthesize, design and develop a wide range of new biologically active compounds.

\section{ACKNOWLEDGEMENT}

We are highly grateful to the Department of Science and Technology (DST), Government of India for the financial support vide their sanction no. 100/(IFD)/1899/2012-13 and Manipal University Jaipur for the other investigation facilities and support.

\section{Conflict of Interest}

The authors have declared no conflict of interest.

\section{References}

[1] Zinner, G. "Lossen, wilhelm-discovery of hydroxylamine 125 years ago (1865)." ChemikerZeitung 114, no. 6 (1990): 197-204.

[2] Szymanowski, J. "Kinetics and interfacial phenomena in solvent extraction of metals." Mineral Processing and Extractive Metullargy Review 18, no. 1 (1998): 166.

[3] Corcuera, Luis J., Michael D. Woodward, John P. Helgeson, Arthur Kelman, and Christen D. Upper. "2, 4Dihydroxy-7-methoxy-2H-1, 4-benzoxazin-3 (4H)-one, an inhibitor from Zea mays with differential activity against soft rotting Erwinia species." Plant Physiology 61, no. 5 (1978): 791-795.

[4] Wang, Ching Yung, and Lanfong H. Lee. "Mutagenicity and antibacterial activity of hydroxamic acids." Antimicrobial agents and chemotherapy 11, no. 4 (1977): 753.

[5] Kobashi, Kyoichi, Kenji Kumaki, and Jun'ichi Hase. "Effect of acyl residues of hydroxamic acids on urease inhibition." Biochimica et Biophysica Acta (BBA)Enzymology 227, no. 2 (1971): 429-441.

[6] Hase, Junichi, Kyoichi Kobashi, Nobuo Kawaguchi, And Kiyonori Sakamoto. "Antimicrobial activity of hydroxamic acids." Chemical and Pharmaceutical Bulletin 19, no. 2 (1971): 363-368.

[7] Kaushal, Raj, Sheetal Thakur, and Kiran Nehra. "CtDNA binding and antibacterial activity of octahedral titanium (IV) heteroleptic (benzoylacetone and hydroxamic acids) complexes." International journal of medicinal chemistry 2016 (2016).

[8] Haron, Md Jelas, et al. "Antifungal properties of phenyl fatty hydroxamic acids and their copper complexes synthesized based on canola and palm kernel oils." Asian J Chem 25 (2013): 4183-4188.

[9] Gleńsk, Michał, Beata Gajda, Roman Franiczek, Barbara Krzyżanowska, Izabela Biskup, and Maciej Włodarczyk. "In vitro evaluation of the antioxidant and antimicrobial activity of DIMBOA [2, 4-dihydroxy-7methoxy-2 H-1, 4-benzoxazin-3 (4 H)-one]." Natural product research 30, no. 11 (2016): 1305-1308.

[10] Albalawi, Marzough. "Evaluation of Antibacterial and Antifungal Effects of Novel Hydroxamic Acids Linkednatural Amino Acids." Egyptian Journal of Chemistry 60, no. 4 (2017): 613-618.

[11] Hidalgo, Manuel, and S. Gail Eckhardt. "Development of matrix metalloproteinase inhibitors in cancer therapy." Journal of the National Cancer Institute 93, no. 3 (2001): 178-193.

[12] Leung, Donmienne, Giovanni Abbenante, and David P. Fairlie. "Protease inhibitors: current status and future prospects." Journal of medicinal chemistry 43, no. 3 (2000): 305-341.

[13] Parker, Matthew H., et al. "Analysis of the binding of hydroxamic acid and carboxylic acid inhibitors to the stromelysin-1 (matrix metalloproteinase-3) catalytic domain by isothermal titration calorimetry." Biochemistry 38, no. 41 (1999): 13592 13601.

[14] Toba, Samuel, K. V. Damodaran, and Kenneth M. Merz. "Binding preferences of hydroxamate inhibitors of the matrix metalloproteinase human fibroblast collagenase." Journal of medicinal chemistry 42, no. 7 (1999): 1225-1234.

[15]Lehmann, Thomas E., and Albrecht Berkessel. "Stereoselective Synthesis of 4 '-BenzophenoneSubstituted Nucleoside Analogs: Photoactive Models for Ribonucleotide Reductases." The Journal of organic chemistry 62, no. 2 (1997): 302-309.

[16]Xie, Kevin Chunxi, and William Plunkett. "Deoxynucleotide pool depletion and sustained inhibition of ribonucleotide reductase and DNA synthesis after treatment of human lymphoblastoid cells with 2-chloro-9-(2-deoxy-2-fluoro- $\beta$-Darabinofuranosyl) adenine." Cancer research 56, no. 13 (1996): 3030-3037.

[17] Salowe, S., et al. "Alternative model for mechanismbased inhibition of Escherichia coli ribonucleotide reductase by 2'-azido-2'-deoxyuridine 5'diphosphate." Biochemistry 32, no. 47 (1993): 12749 12760

[18] Agarwal, Hemlata, O. P. Agarwal, R. Karnawat, I. K. Sharma, and P. S. Verma. "Synthesis, characterisation and biocidal studies of some hydroxamic acids." (2010).

[19] Davis, Alvie L., Karen L. Hulme, Gerald T. Wilson, and Tommy J. Mccord. "In vitro antimicrobial activity of some cyclic hydroxamic acids and related lactams." Antimicrobial agents and chemotherapy 13, no. 3 (1978): 542-544.

[20] Sato, Ayami, et al. "Suppressive effect of the histone deacetylase inhibitor suberoylanilide hydroxamic acid (SAHA) on hepatitis C virus replication." Journal of cellular biochemistry 114, no. 9 (2013): 1987-1996.

[21] Saha, Bratati, and Robin J. Parks. "Histone deacetylase inhibitor suberoylanilide hydroxamic acid suppresses human adenovirus gene expression and replication." Journal of virology 93, no. 12 (2019): e00088-19. 
[22] Apfel, Christian, et al. "Hydroxamic acid derivatives as potent peptide deformylase inhibitors and antibacterial agents." Journal of medicinal chemistry 43, no.12 (2000): 2324-2331.

[23] Meinnel, Thierry, Christine Lazennec, Stéphane Villoing, and Sylvain Blanquet. "Structure-function relationships within the peptide deformylase family. Evidence for a conserved architecture of the active site involving three conserved motifs and a metal ion." Journal of molecular biology 267, no. 3 (1997): 749-761.

[24] Rasmussen, Henrik S., and Peter P. McCann. "Matrix metalloproteinase inhibition as a novel anticancer strategy: a review with special focus on batimastat and marimastat." Pharmacology \& therapeutics 75 , no. 1 (1997): 69-75.

[25] Chen, Dawn Z., et al. "Actinonin, a naturally occurring antibacterial agent, is a potent deformylase inhibitor." Biochemistry 39, no. 6 (2000): 1256-1262.

[26] Yamamoto, Minoru, et al. "Inhibition of membrane-type 1 matrix metalloproteinase by hydroxamate inhibitors: an examination of the subsite pocket." Journal of medicinal chemistry 41, no. 8 (1998): 1209-1217.

[27] Gao, Jian, et al. "Design, Synthesis, and Biological Evaluation of Vanillin Hydroxamic Acid Derivatives as Novel Peptide Deformylase Inhibitors." Current computer-aided drug design 14, no.1 (2018): 95-101.

[28] Clements, John M., et al. "Antibiotic activity and characterization of BB-3497, a novel peptide deformylase inhibitor." Antimicrobial agents and chemotherapy 45, no. 2 (2001): 563-570.

[29] Alkiewicz, J., Z. Eckstein, H. Halweg, P. Krakowka, and T. Urbański. "Fungistatic activity of some hydroxamic acids." Nature 180, no. 4596 (1957): 12041205.

[30] Flynn, Daniel L., et al. "Nonsteroidal anti-inflammatory drug hydroxamic acids. Dual inhibitors of both cyclooxygenase and 5-lipoxygenase." Journal of medicinal chemistry 33, no. 8 (1990): 2070-2072.

[31]Fiorucci, Stefano, Rosaria Meli, Mariarosaria Bucci, and Giuseppe Cirino. "Dual inhibitors of cyclooxygenase and 5-lipoxygenase. A new avenue in anti-inflammatory therapy" Biochemical pharmacology 62, no. 11 (2001): 1433-1438.

[32]Pontiki, Eleni, and Dimitra Hadjipavlou-Litina. "Antioxidant and anti-inflammatory activity of arylacetic and hydroxamic acids as novel lipoxygenase inhibitors." Medicinal Chemistry 2, no. 3 (2006): 251 264.

[33]Lohman, Rink-Jan, et al. "Differential antiinflammatory activity of HDAC inhibitors in human macrophages and rat arthritis." Journal of Pharmacology and Experimental Therapeutics 356, no. 2 (2016): 387-396.

[34] Chen, Hui, Jing Pan, Jin-dan Wang, Qiu-mei Liao, and Xiao-ru Xia. "Suberoylanilide hydroxamic acid, an inhibitor of histone deacetylase, induces apoptosis in rheumatoid arthritis fibroblast-like synoviocytes." Inflammation 39, no. 1 (2016): 39-46.
[35] Eckschlager, Tomas, Johana Plch, Marie Stiborova, and Jan Hrabeta. "Histone deacetylase inhibitors as anticancer drugs." International journal of molecular sciences 18, no. 7 (2017): 1414.

[36] Niemeyer, Hermann M., Erika Pesel, Sylvia V. Copaja, Héctor R. Bravo, Stephan Franke, and Wittko Francke. "Changes in hydroxamic acid levels of wheat plants induced by aphid feeding." Phytochemistry 28, no. 2 (1989): 447-449.

[37] Friebe, Annette, Udo Roth, Peter Kück, Heide Schnabl, and Margot Schulz. "Effects of 2, 4-dihydroxy-1, 4benzoxazin-3-ones on the activity of plasma membrane H+-ATPase." Phytochemistry 44, no. 6 (1997): 979983.

[38] Massardo, Francisca, Gustavo E. Zúñiga, Luz M. Ptrez, and Luis J. Corcuerat. "Effects of hydroxamic acids on electron transport and their cellular location in corn." Phytochemistry 35, no. 4 (1994): 873-876.

[39] Duvic, Madeleine, and Jenny Vu. "Vorinostat: a new oral histone deacetylase inhibitor approved for cutaneous T-cell lymphoma." Expert opinion on investigational drugs 16, no. 7 (2007): 1111-1120.

[40]Richon, V. M. "Cancer biology: mechanism of antitumour action of vorinostat (suberoylanilide hydroxamic acid), a novel histone deacetylase inhibitor." British journal of cancer 95, no. 1 (2006): S2-S6.

[41] Finnin, Michael S., et al. "Structures of a histone deacetylase homologue bound to the TSA and SAHA inhibitors." Nature 401, no. 6749 (1999): 188-193.

[42] Marks, Paul A., Victoria M. Richon, and Richard A. Rifkind. "Histone deacetylase inhibitors: inducers of differentiation or apoptosis of transformed cells." Journal of the National Cancer Institute 92, no. 15 (2000): 1210-1216.

[43] Fischle, Wolfgang, Yanming Wang, and C. David Allis. "Binary switches and modification cassettes in histone biology and beyond." Nature 425, no. 6957 (2003): 475-479.

[44] Jenuwein, Thomas, and C. David Allis. "Translating the histone code." Science 293, no. 5532 (2001): 10741080 .

[45] Spotswood, Hugh T., and Bryan M. Turner. "An increasingly complex code." The Journal of clinical investigation 110, no. 5 (2002): 577-582.

[46]Zhang, Chunlei, Victoria Richon, Xiao Ni, Rakhshandra Talpur, and Madeleine Duvic. "Selective induction of apoptosis by histone deacetylase inhibitor SAHA in cutaneous T-cell lymphoma cells: relevance to mechanism of therapeutic action." Journal of Investigative Dermatology 125, no. 5 (2005): 10451052.

[47]Huang, Lili, and Arthur B. Pardee. "Suberoylanilide hydroxamic acid as a potential therapeutic agent for human breast cancer treatment." Molecular medicine 6, no. 10 (2000): 849-866.

[48] Tong, Aiping, et al. "Proteomic analysis of liver cancer cells treated with suberonylanilide hydroxamic acid." Cancer chemotherapy and pharmacology 61, no. 5 (2008): 791-802. 
[49] Seo, Sung-Keum, et al. "Combined effects of sulindac and suberoylanilide hydroxamic acid on apoptosis induction in human lung cancer cells." Molecular pharmacology 73, no. 3 (2008): 1005-1012.

[50] Ling, Yong, et al. "Novel $\beta$-carboline/hydroxamic acid hybrids targeting both histone deacetylase and DNA display high anticancer activity via regulation of the p53 signaling pathway." Journal of medicinal chemistry 58, no. 23 (2015): 9214-9227.

[51]Chen, Jiwei, et al. "Design, synthesis and anticancer evaluation of acridine hydroxamic acid derivatives as dual Topo and HDAC inhibitors." Bioorganic \& medicinal chemistry 26, no. 14 (2018): 3958-3966.

[52] Gora-Tybor, J., and T. Robak. "Clinical pharmacology of hydroxyurea." Acta haematologica Polonica 26, no. 1 (1995): 39-45.

[53]Elford, Howard L., Galen L. Wampler, and Bart van't Riet. "New ribonucleotide reductase inhibitors with antineoplastic activity." Cancer research 39, no. 3 (1979): 844-851.

[54] Sur, P., S. P. Bag, B. Sur, and J. A. Khanam. "Chloroaceto hydroxamic acid as antitumor agent against Ehrlich ascites carcinoma in mice." Neoplasma 44, no. 3 (1997): 197-201.

[55] Tsukamoto, Kikuo, et al. "Binding of salicylhydroxamic acid and several aromatic donor molecules to Arthromyces ramosus peroxidase, investigated by X-ray crystallography, optical difference spectroscopy, NMR relaxation, molecular dynamics, and kinetics." Biochemistry 38, no. 39 (1999): 12558-12568.

[56] Szekeres, Thomas, Monika Fritzer-Szekeres, Howard L. Elford, and H. M. Jayaram. "The enzyme ribonucleotide reductase: target for antitumor and anti-HIV therapy." Critical reviews in clinical laboratory sciences 34, no. 6 (1997): 503-528.

[57] Giannini, Giuseppe, Gianfranco Battistuzzi, and Davide Vignola. "Hydroxamic acid based histone deacetylase inhibitors with confirmed activity against the malaria parasite." Bioorganic \& medicinal chemistry letters 25, no. 3 (2015): 459-461.

[58] Diedrich, Daniela, et al. "One-pot, multi-component synthesis and structure-activity relationships of peptoidbased histone deacetylase (HDAC) inhibitors targeting malaria parasites." European journal of medicinal chemistry 158 (2018): 801-813.

[59] Chance, Jeffrey P., et al. "Development of piperazinebased hydroxamic acid inhibitors against falcilysin, an essential malarial protease." Bioorganic \& medicinal chemistry letters 28, no. 10 (2018): 1846-1848.

[60] Cabantchik, Ioav, Hava Glickstein, Jacob Golenser, Mark Loyevsky, and Appolinaire Tsafack. "Iron chelators: mode of action as antimalarials." Acta haematologica 95, no. 1 (1996): 70-77.

[61] Mishra, Hetal, Abby L. Parrill, and John S. Williamson. "Three-dimensional quantitative structure-activity relationship and comparative molecular field analysis of dipeptide hydroxamic acid Helicobacter pylori urease inhibitors." Antimicrobial agents and chemotherapy 46, no. 8 (2002): 2613-2618.
[62] Rammohan, Aluru, Julakanti Satyanarayana Reddy, Gundala Sravya, Chittluri Narasimha Rao, and Grigory V. Zyryanov. "Chalcone synthesis, properties and medicinal applications: a review." Environmental Chemistry Letters (2020): 1-26.

[63] Rao, Martin, Davide Valentini, Alimuddin Zumla, and Markus Maeurer. "Evaluation of the efficacy of valproic acid and suberoylanilide hydroxamic acid (vorinostat) in enhancing the effects of first-line tuberculosis drugs against intracellular Mycobacterium tuberculosis." International Journal of Infectious Diseases 69 (2018): 78-84.

[64] Miller, H. E., F. Rigelhof, L. Marquart, A. Prakash, and M. Kanter. "Whole-grain products and antioxidants." Cereal foods world 45, no. 2 (2000): 59 63.

[65]Luo, Meng, et al. "Cajanol, a novel anticancer agent from Pigeonpea [Cajanus cajan (L.) Millsp.] roots, induces apoptosis in human breast cancer cells through a ROS-mediated mitochondrial pathway." ChemicoBiological Interactions 188, no. 1 (2010): 151-160.

[66]Conner, Elaine M., and Matthew B. Grisham. "Inflammation, free radicals, and antioxidants." Nutrition 12, no. 4 (1996): 274-277.

[67] Trueba, Gilberto Pérez, Gregorio Martínez Sánchez, and Atilia Giuliani. "Oxygen free radical and antioxidant defense mechanism in cancer." Front Biosci 9, no. Sep 1 (2004): 2029-2044.

[68] Ratnam, D. Venkat, D. D. Ankola, V. Bhardwaj, D. Kumar Sahana, and MNV Ravi Kumar. "Role of antioxidants in prophylaxis and therapy: A pharmaceutical perspective." Journal of controlled release 113, no. 3 (2006): 189-207.

[69] Končič, Marijana, Zrinka Rajič, Neva Petrič, and Branka Zorc. "Antioxidant activity of NSAID hydroxamic acids." Acta pharmaceutica 59, no. 2 (2009): 235-242.

[70] Shao, Le-Le, et al. "Preparation, antioxidant and antimicrobial evaluation of hydroxamated degraded polysaccharides from Enteromorpha prolifera." Food chemistry 237 (2017): 481-487.

[71] Li, Yin-Ting, et al. "Antioxidant and antimicrobial evaluation of carboxymethylated and hydroxamated degraded polysaccharides from Sargassum fusiforme." International journal of biological macromolecules 118 (2018): 1550-1557.

\section{Creative Commons Attribution License 4.0 (Attribution 4.0 International, CC BY 4.0)}

This article is published under the terms of the Creative Commons Attribution License 4.0 https://creativecommons.org/licenses/by/4.0/deed.en_US 